\title{
On the Approximation of the Cut and Step Functions by Logistic and Gompertz Functions
}

\author{
Anton Iliev $^{* \dagger}$, Nikolay Kyurkchiev ${ }^{\dagger}$, Svetoslav Markov ${ }^{\dagger}$ \\ *Faculty of Mathematics and Informatics \\ Paisii Hilendarski University of Plovdiv, Plovdiv, Bulgaria \\ Email: aii@uni-plovdiv.bg \\ ${ }^{\dagger}$ Institute of Mathematics and Informatics \\ Bulgarian Academy of Sciences, Sofia, Bulgaria \\ Emails: nkyurk@math.bas.bg, smarkov@bio.bas.bg
}

Received: , accepted: , published: will be added later

\begin{abstract}
We study the uniform approximation of the sigmoid cut function by smooth sigmoid functions such as the logistic and the Gompertz functions. The limiting case of the interval-valued step function is discussed using Hausdorff metric. Various expressions for the error estimates of the corresponding uniform and Hausdorff approximations are obtained. Numerical examples are presented using CAS MATHEMATICA.
\end{abstract}

Keywords-cut function; step function; sigmoid function; logistic function; Gompertz function; squashing function; Hausdorff approximation.

\section{INTRODUCTION}

In this paper we discuss some computational, modelling and approximation issues related to several classes of sigmoid functions. Sigmoid functions find numerous applications in various fields related to life sciences, chemistry, physics, artificial intelligence, etc. In fields such as signal processing, pattern recognition, machine learning, artificial neural networks, sigmoid functions are also known as "activation" and "squashing" functions. In this work we concentrate on several practically important classes of sigmoid functions. Two of them are the cut (or ramp) functions and the step functions. Cut functions are continuous but they are not smooth (differentiable) at the two endpoints of the interval where they increase. Step functions can be viewed as limiting case of cut functions; they are not continuous but they are Hausdorff continuous (H-continuous) [4], [43]. In some applications smooth sigmoid functions are preferred, some authors even require smoothness in the definition of sigmoid functions. Two familiar classes of smooth sigmoid functions are the logistic and the Gompertz functions. There are situations when one needs to pass from nonsmooth sigmoid functions (e. g. cut functions) to smooth sigmoid functions, and vice versa. Such a necessity rises the issue of approximating nonsmooth sigmoid functions by smooth sigmoid functions.

One can encounter similar approximation problems when looking for appropriate models for fitting time course measurement data coming e. g. from cellular growth experiments. Depending on the general view of the data one can decide to use 
initially a cut function in order to obtain rough initial values for certain parameters, such as the maximum growth rate. Then one can use a more sophisticate model (logistic or Gompertz) to obtain a better fit to the measurement data. The presented results may be used to indicate to what extend and in what sense a model can be improved by another one and how the two models can be compared.

Section 2 contains preliminary definitions and motivations. In Section 3 we study the uniform and Hausdorff approximation of the cut functions by logistic functions. Curiously, the uniform distance between a cut function and the logistic function of best uniform approximation is an absolute constant not depending on the slope of the functions, a result observed in [18]. By contrast, it turns out that the Hausdorff distance (H-distance) depends on the slope and tends to zero when increasing the slope. Showing that the family of logistic functions cannot approximate the cut function arbitrary well, we then consider the limiting case when the cut function tends to the step function (in Hausdorff sense). In this way we obtain an extension of a previous result on the Hausdorff approximation of the step function by logistic functions [4]. In Section 4 we discuss the approximation of the cut function by a family of squashing functions induced by the logistic function. It has been shown in [18] that the latter family approximates uniformly the cut function arbitrary well. We propose a new estimate for the $\mathrm{H}$-distance between the cut function and its best approximating squashing function. Our estimate is then extended to cover the limiting case of the step function. In Section 5 the approximation of the cut function by Gompertz functions is considered using similar techniques as in the previous sections. The application of the logistic and Gompertz functions in life sciences is briefly discussed. Numerical examples are presented throughout the paper using the computer algebra system MATHEMATICA.

\section{PRELIMINARIES}

Sigmoid functions. In this work we consider sigmoid functions of a single variable defined on the real line, that is functions $s$ of the form $s: \mathbb{R} \longrightarrow \mathbb{R}$. Sigmoid functions can be defined as bounded monotone non-decreasing functions on $\mathbb{R}$. One usually makes use of normalized sigmoid functions defined as monotone non-decreasing functions $s(t), t \in \mathbb{R}$, such that $\lim s(t)_{t \rightarrow-\infty}=0$ and $\lim s(t)_{t \rightarrow \infty}=1$. In the fields of neural networks and machine learning sigmoid-like functions of many variables are used, familiar under the name activation functions. (In some applications the sigmoid functions are normalised so that the lower asymptote is assumed $-1: \lim s(t)_{t \rightarrow-\infty}=$ -1$.

Cut (ramp) functions. Let $\Delta=[\gamma-\delta, \gamma+\delta]$ be an interval on the real line $\mathbb{R}$ with centre $\gamma \in \mathbb{R}$ and radius $\delta \in \mathbb{R}$. A cut function (on $\Delta$ ) is defined as follows:

Definition 1. The cut function $c_{\gamma, \delta}$ on $\Delta$ is defined for $t \in \mathbb{R}$ by

$$
c_{\gamma, \delta}(t)=\left\{\begin{array}{cl}
0, & \text { if } t<\Delta, \\
\frac{t-\gamma+\delta}{2 \delta}, & \text { if } t \in \Delta, \\
1, & \text { if } \Delta<t .
\end{array}\right.
$$

Note that the slope of function $c_{\gamma, \delta}(t)$ on the interval $\Delta$ is $1 /(2 \delta)$ (the slope is constant in the whole interval $\Delta$ ). Two special cases are of interest for our discussion in the sequel.

Special case 1. For $\gamma=0$ we obtain a cut function on the interval $\Delta=[-\delta, \delta]$ :

$$
c_{0, \delta}(t)=\left\{\begin{array}{cll}
0, & \text { if } \quad t<-\delta, \\
\frac{t+\delta}{2 \delta}, & \text { if } \quad-\delta \leq t \leq \delta, \\
1, & \text { if } \quad \delta<t .
\end{array}\right.
$$

Special case 2. For $\gamma=\delta$ we obtain the cut function on $\Delta=[0,2 \delta]$ :

$$
c_{\delta, \delta}(t)=\left\{\begin{array}{cll}
0, & \text { if } & t<0 \\
\frac{t}{2 \delta}, & \text { if } & 0 \leq t \leq 2 \delta \\
1, & \text { if } & 2 \delta<t
\end{array}\right.
$$


Step functions. The step function (with "jump" at $\gamma \in \mathbb{R})$ can be defined by

$$
h_{\gamma}(t)=c_{\gamma, 0}(t)=\left\{\begin{array}{cl}
0, & \text { if } t<\gamma, \\
{[0,1],} & \text { if } t=\gamma \\
1, & \text { if } t>\gamma
\end{array}\right.
$$

which is an interval-valued function (or just interval function) [4], [43]. In the literature various point values, such as $0,1 / 2$ or 1 , are prescribed to the step function (4) at the point $\gamma$; we prefer the interval value $[0,1]$. When the jump is at the origin, that is $\gamma=0$, then the step function is known as the Heaviside step function; its "interval" formulation is:

$$
h_{0}(t)=c_{0,0}(t)=\left\{\begin{array}{cc}
0, & \text { if } t<0 \\
{[0,1],} & \text { if } t=0 \\
1, & \text { if } t>0
\end{array}\right.
$$

H-distance. The step function can be perceived as a limiting case of the cut function. Namely, for $\delta \rightarrow 0$, the cut function $c_{\delta, \delta}$ tends in "Hausdorff sense" to the step function. Here "Hausdorff sense" means Hausdorff distance, briefly $H$-distance. The H-distance $\rho(f, g)$ between two interval functions $f, g$ on $\Omega \subseteq \mathbb{R}$, is the distance between their completed graphs $F(f)$ and $F(g)$ considered as closed subsets of $\Omega \times \mathbb{R}[24]$, [41]. More precisely,

$$
\begin{gathered}
\rho(f, g)=\max \left\{\sup _{A \in F(f)} \inf _{B \in F(g)}\|A-B\|,\right. \\
\left.\sup _{B \in F(g)} \inf _{A \in F(f)}\|A-B\|\right\},
\end{gathered}
$$

wherein $\|$.$\| is any norm in \mathbb{R}^{2}$, e. g. the maximum norm $\|(t, x)\|=\max |t|,|x|$.

To prove that (3) tends to (5) let $h$ be the $\mathrm{H}$ distance between the step function (5) and the cut function (3) using the maximum norm, that is a square (box) unit ball. By definition (6) $h$ is the side of the smallest unit square, centered at the point $(0,1)$ touching the graph of the cut function. Hence we have $1-c_{\delta, \delta}(h)=h$, that is $1-h /(2 \delta)=h$, implying

$$
h=\frac{2 \delta}{1+2 \delta}=2 \delta+O\left(\delta^{2}\right) .
$$

For the sake of simplicity throughout the paper we shall work with some of the special cut functions (2), (3), instead of the more general (arbitrary shifted) cut function (1); these special choices will not lead to any loss of generality concerning the results obtained. Moreover, for all sigmoid functions considered in the sequel we shall define a "basic" sigmoid function such that any member of the corresponding class is obtained by replacing the argument $t$ by $t-\gamma$, that is by shifting the basic function by some $\gamma \in \mathbb{R}$.

\section{Logistic and Gompertz functions: applications}

to life-sciences. In this work we focus on two familiar smooth sigmoid functions, namely the Gompertz function and the Verhulst logistic function. Both their inventors, B. Gompertz and P.F. Verhulst, have been motivated by the famous demographic studies of Thomas Malthus.

The Gompertz function was introduced by Benjamin Gompertz [22] for the study of demographic phenomena, more specifically human aging [38], [39], [47]. Gompertz functions find numerous applications in biology, ecology and medicine. A. K. Laird successfully used the Gompertz curve to fit data of growth of tumors [32]; tumors are cellular populations growing in a confined space where the availability of nutrients is limited [1], [2], [15], [19].

A number of experimental scientists apply Gompertz models in bacterial cell growth, more specifically in food control [10], [31], [42], [48], [49], [50]. Gompertz models prove to be useful in animal and agro-sciences as well [8], [21], [27], [48]. The Gompertz model has been applied in modelling aggregation processes [25], [26]; it is a subject of numerous theoretical modelling studies as well [6], [7], [9], [20], [37], [40].

The logistic function was introduced by Pierre François Verhulst [44]-[46], who applied it to human population dynamics. Verhulst derived his logistic equation to describe the mechanism of the self-limiting growth of a biological population. The equation was rediscovered in 1911 by $\mathrm{A}$. G. McKendrick [35] for the bacterial growth in 
broth and was tested using nonlinear parameter estimation. The logistic function finds applications in an wide range of fields, including biology, ecology, population dynamics, chemistry, demography, economics, geoscience, mathematical psychology, probability, sociology, political science, financial mathematics, statistics, fuzzy set theory, to name a few [12], [13], [11], [14], [18].

Logistic functions are often used in artificial neural networks [5], [16], [17], [23]. Any neural net element computes a linear combination of its input signals, and applies a logistic function to the result; often called "activation" function. Another application of logistic curve is in medicine, where the logistic differential equation is used to model the growth of tumors. This application can be considered an extension of the above-mentioned use in the framework of ecology. In (bio)chemistry the concentration of reactants and products in autocatalytic reactions follow the logistic function.

Other smooth sigmoid functions. The integral (antiderivative) of any smooth, positive, "bumpshaped" or "bell-shaped" function will be sigmoidal. A famous example is the error function, which is the integral (also called the cumulative distribution function) of the Gaussian normal distribution. The logistic function is also used as a base for the derivation of other sigmoid functions, a notable example is the generalized logistic function, also known as Richards curve [37]. Another example is the Dombi-Gera-squashing function introduced and studied in [18] obtained as an antiderivative (indefinite integral) of the difference of two shifted logistic functions.

In what follows we shall be interested in the approximation of the cut function by smooth sigmoid functions, more specifically the Gompertz, the logistic and the Dombi-Gera-squashing function. We shall focus first on the Verhulst logistic function.

\section{Approximation OF THE CUT FUNCTION BY LOGISTIC FUNCTIONS}

Definition 2. Define the logistic (Verhulst) function $v$ on $\mathbb{R}$ as [44]-[46]

$$
v_{\gamma, k}(t)=\frac{1}{1+e^{-4 k(t-\gamma)}} .
$$

Note that the logistic function (7) has an inflection at its "centre" $(\gamma, 1 / 2)$ and its slope at $\gamma$ is equal to $k$.

Proposition 1. [18] The function $v_{\gamma, k}(t)$ defined by (7) with $k=1 /(2 \delta)$ : i) is the logistic function of best uniform one-sided approximation to function $c_{\gamma, \delta}(t)$ in the interval $[\gamma, \infty)$ (as well as in the interval $(-\infty, \gamma])$; ii) approximates the cut function $c_{\gamma, \delta}(t)$ in uniform metric with an error

$$
\rho=\rho(c, v)=\frac{1}{1+e^{2}}=0.11920292 \ldots
$$

Proof. Consider functions (1) and (7) with same centres $\gamma=\delta$, that is functions $c_{\delta, \delta}$ and $v_{\delta, k}$. In addition chose $c$ and $v$ to have same slopes at their coinciding centres, that is assume $k=1 /(2 \delta)$, cf. Figure 1. Then, noticing that the largest uniform distance between the cut and logistic functions is achieved at the endpoints of the underlying interval $[0,2 \delta]$, we have:

$$
\rho=v_{\delta, k}(0)-c_{\delta, \delta}=\frac{1}{1+e^{4 k \delta}}=\frac{1}{1+e^{2}} .
$$

This completes the proof of the proposition.

We note that the uniform distance (9) is an absolute constant that does not depend on the width of the underlying interval $\Delta$, resp. on the slope $k$. The next proposition shows that this is not the case whenever H-distance is used.

Proposition 2. The function $v(t)=v_{0, k}(t)$ with $k=1 /(2 \delta)$ is the logistic function of best Hausdorff one-sided approximation to function $c(t)=$ $c_{0, k}(t)$ in the interval $[0, \infty)$ (resp. in the interval $(-\infty, 0])$. The function $v(t)$, approximates function $c(t)$ in H-distance with an error $h=h(c, v)$ that satisfies the relation:

$$
\ln \frac{1-h}{h}=2+4 k h \text {. }
$$


A. Iliev et al., On the Approximation of the Cut and Step Functions by Logistic ...

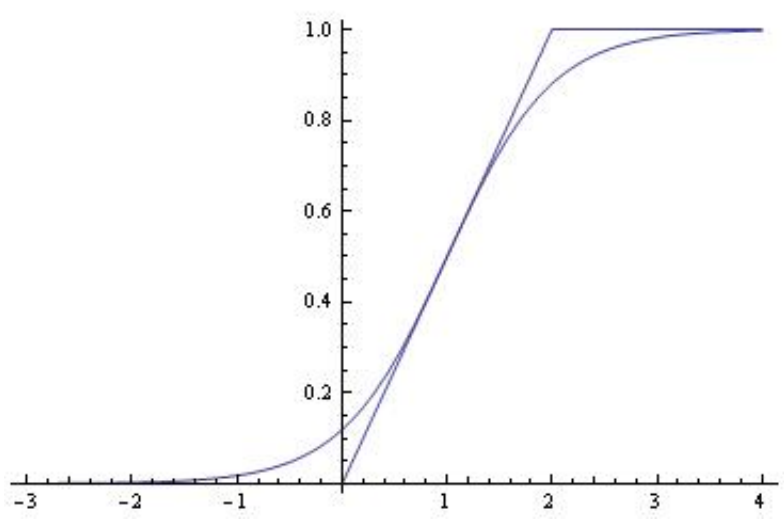

Fig. 1. The cut and logistic functions for $\gamma=\delta=1$, $k=1 / 2$.

Proof. Using $\delta=1 /(2 k)$ we can write $\delta+h=$ $(1+2 h k) /(2 k)$, resp.:

$$
v(-\delta-h)=\frac{1}{1+e^{2(1+2 h k)}}
$$

The H-distance $h$ using square unit ball (with a side $h$ ) satisfies the relation $v(-\delta-h)=h$, which implies (10). This completes the proof of the proposition.

Relation (10) shows that the H-distance $h$ depends on the slope $k, h=h(k)$. The next result gives additional information on this dependence.

Proposition 3. For the H-distance $h(k)$ the following holds for $k>5$ :

$$
\frac{1}{4 k+1}<h(k)<\frac{\ln (4 k+1)}{4 k+1} .
$$

Proof. We need to express $h$ in terms of $k$, using (10). Let us examine the function

$$
f(h)=2+4 h k-\ln (1-h)-\ln \frac{1}{h} .
$$

From

$$
f^{\prime}(h)=4 k+\frac{1}{1-h}+\frac{1}{h}>0
$$

we conclude that function $f$ is strictly monotone increasing. Consider the function

$$
g(h)=2+h(1+4 k)-\ln \frac{1}{h} .
$$

Then $g(h)-f(h)=h+\ln (1-h)=O\left(h^{2}\right)$ using the Taylor expansion $\ln (1-h)=-h+O\left(h^{2}\right)$. Hence $g(h)$ approximates $f(h)$ with $h \rightarrow 0$ as $O\left(h^{2}\right)$. In addition $g^{\prime}(h)=1+4 k+1 / h>0$, hence function $g$ is monotone increasing. Further, for $k \geq 5$

$$
\begin{aligned}
g\left(\frac{1}{1+4 k}\right) & =3-\ln (1+4 k)<0, \\
g\left(\frac{\ln (4 k+1)}{4 k+1}\right) & =2+\ln \ln (1+4 k)>0 .
\end{aligned}
$$

This completes the proof of the proposition.

Relation (11) implies that when the slope $k$ of functions $c$ and $v$ tends to infinity, the h-distance $h(c, v)$ between the two functions tends to zero (differently to the uniform distance $\rho(c, v)$ which remains constant).

The following proposition gives more precise upper and lower bounds for $h(k)$. For brevity denote $K=4 k+1$.

Proposition 4. For the H-distance $h$ the following inequalities hold for $k \geq 5$ :

$$
\begin{gathered}
\frac{\ln K}{K}-\frac{2+\ln \ln K}{K\left(1+\frac{1}{\ln K}\right)}<h(k)< \\
\frac{\ln K}{K}+\frac{2+\ln \ln K}{K\left(\frac{\ln \ln K}{1-\ln K}-1\right)}, K=4 k+1 .
\end{gathered}
$$

Proof. Evidently, the second derivative of $g(h)=$ $2+h(1+4 k)-\ln (1 / h)$, namely $g^{\prime \prime}(h)=$ $-\frac{1}{h^{2}}<0$, has a constant sign on $\left[\frac{1}{K}, \frac{\ln K}{K}\right]$. The straight line, defined by the points $\left(\frac{1}{K}, g\left(\frac{1}{K}\right)\right)$ and $\left(\frac{\ln K}{K}, g\left(\frac{\ln K}{K}\right)\right)$, and the tangent to $g$ at the point $\left(\frac{\ln K}{K}, g\left(\frac{\ln K}{K}\right)\right)$ cross the abscissa at the points

$\frac{\ln K}{K}+\frac{2+\ln \ln K}{K\left(\frac{\ln \ln K}{1-\ln K}-1\right)}, \frac{\ln K}{K}-\frac{2+\ln \ln K}{K\left(1+\frac{1}{\ln K}\right)}$, respectively. This completes the proof of the Proposition.

Propositions 2, 3 and 4 extend similar results from [4] stating that the Heaviside interval-valued step function is approximated arbitrary well by 
A. Iliev et al., On the Approximation of the Cut and Step Functions by Logistic ...

logistic functions in Hausdorff metric. The Hausdorff approximation of the Heaviside step function by sigmoid functions is discussed from various computational and modelling aspects in [28], [29], [30].

\section{APPROXIMATION OF THE CUT FUNCTION BY A SQUASHING FUNCTION}

The results obtained in Section 3 state that the cut function cannot be approximated arbitrary well by the family of logistic functions. This result justifies the discussion of other families of smooth sigmoid functions having better approximating properties. Such are the squashing functions proposed in [18] further denoted DGsquashing functions.

Definition 3. The DG-squashing function $s_{\Delta}$ on the interval $\Delta=[\gamma-\delta, \gamma+\delta]$ is defined by

$$
s_{\Delta}^{(\beta)}(t)=s_{\gamma, \delta}^{(\beta)}(t)=\frac{1}{2 \delta} \ln \left(\frac{1+e^{\beta(t-\gamma+\delta)}}{1+e^{\beta(t-\gamma-\delta)}}\right)^{\frac{1}{\beta}} .
$$

Note that the squashing function (13) has an inflection at its "centre" $\gamma$ and its slope at $\gamma$ is equal to $(2 \delta)^{-1}$.

The squashing function (13) with centre $\gamma=\delta$ :

$$
s_{\delta, \delta}^{(\beta)}(t)=\frac{1}{2 \delta} \ln \left(\frac{1+e^{\beta t}}{1+e^{\beta(t-2 \delta)}}\right)^{\frac{1}{\beta}},
$$

is the function of best uniform approximation to the cut function 3 . Indeed, functions $c_{\delta, \delta}$ and $s_{\gamma, \delta}^{(\beta)}$ have same centre $\gamma=\delta$ and equal slopes $1 /(2 \delta)$ at their coinciding centres. As in the case with the logistic function, one observes that the uniform distance $\rho=\rho(c, s)$ between the cut and squashing function is achieved at the endpoints of the interval $\Delta$, more specifically at the origin. Denoting the width of the interval $\Delta$ by $w=2 \delta$ we obtain

$$
\begin{gathered}
\rho=s_{\delta, \delta}^{(\beta)}(0)=\frac{1}{w} \ln \left(\frac{2}{1+e^{\beta(-w)}}\right)^{1 / \beta}< \\
\frac{\ln 2}{w} \frac{1}{\beta}=\mathrm{const} \frac{1}{\beta} .
\end{gathered}
$$

The estimate 15 has been found by Dombi and Gera [18]. This result shows that any cut

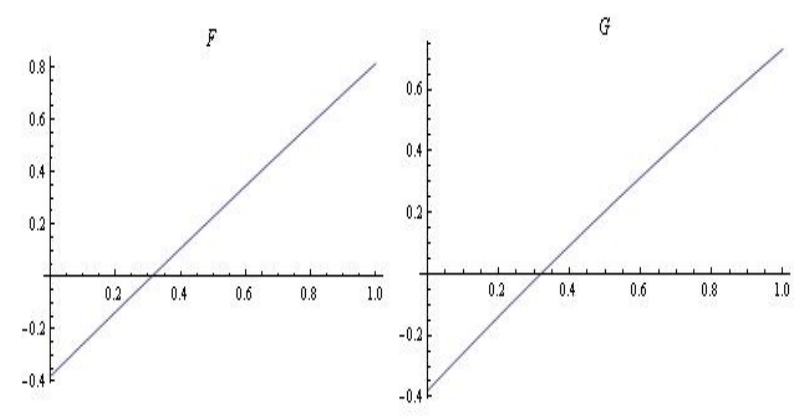

Fig. 2. The functions $F(d)$ and $G(d)$.

function $c_{\Delta}$ can be approximated arbitrary well by squashing functions $s_{\Delta}^{(\beta)}$ from the class 13 . The approximation becomes better with the increase of the value of the parameter $\beta$. Thus $\beta$ affects the quality of the approximation; as we shall see below the practically interesting values of $\beta$ are integers greater than 4 .

In what follows we aim at an analogous result using Hausdorff distance. Let us fix again the centres of the cut and squashing functions to be $\gamma=\delta$ so that the form of the cut function is $c_{\delta, \delta}$, namely (3), whereas the form of the squashing function is $s_{\delta, \delta}^{(\beta)}$ as given by 14 . Both functions $c_{\delta, \delta}$ and $s_{\delta, \delta}^{(\beta)}$ have equal slopes $1 / w, w=2 \delta$, at their centres $\delta$.

Denoting the square-based H-distance between $c_{\delta, \delta}$ and $s_{\delta, \delta}^{(\beta)}$ by $d=d(w ; \beta), w=2 \delta$, we have the relation

$$
s_{\delta, \delta}^{(\beta)}(w+d)=\frac{1}{w} \ln \left(\frac{1+e^{\beta(w+d)}}{1+e^{\beta d}}\right)^{\frac{1}{\beta}}=1-d
$$

or

$$
\ln \frac{1+e^{\beta(w+d)}}{1+e^{\beta d}}=\beta w(1-d)
$$

The following proposition gives an upper bound for $d=d(w ; \beta)$ as implicitly defined by $(16)$ :

Proposition 5. For the distance $d$ the following holds for $\beta \geq 5$ :

$$
d<\ln 2 \frac{\ln (4 \beta w+1)}{4 w \beta+1} .
$$


A. Iliev et al., On the Approximation of the Cut and Step Functions by Logistic ...

Proof. We examine the function:

$F(d)=-\beta w(1-d)+\ln \left(1+e^{\beta(w+d)}\right)+\ln \frac{1}{1+e^{\beta d}}$.

From $F^{\prime}(d)>0$ we conclude that function $F(d)$ is strictly monotone increasing. We define the function

$$
\begin{gathered}
G(d)=-\beta w+\ln \left(1+e^{\beta w}\right)+ \\
d \beta\left(w+\frac{e^{\beta w}}{1+e^{\beta w}}\right)+\ln \frac{1}{1+e^{\beta d}} .
\end{gathered}
$$

We examine $G(d)-F(d)$ :

$$
\begin{aligned}
& G(d)-F(d)= \\
& \quad \ln \left(1+e^{\beta w}\right)+\frac{e^{\beta w} \beta d}{1+e^{\beta w}}-\ln \left(1+e^{\beta(w+d)}\right) .
\end{aligned}
$$

From Taylor expansion

$\ln \left(1+e^{\beta(w+d)}\right)=\ln \left(1+e^{\beta w}\right)+\frac{e^{\beta w} \beta d}{1+e^{\beta w}}+O\left(d^{2}\right)$

we see that function $G(d)$ approximates $F(d)$ with $d \rightarrow 0$ as $O\left(d^{2}\right)$ (cf. Fig. 2).

In addition $G(0)<0$ and $G\left(\ln 2 \frac{\ln (4 \beta w+1)}{4 w \beta+1}\right)>$ 0 for $\beta \geq 5$. This completes the proof of the proposition.

Some computational examples using relation (16) and (17) for various $\beta$ and $w$ are presented in Table 1.

\begin{tabular}{|c||c||c|c|}
\hline$w$ & $\beta$ & $d(w ; \beta)$ from $\sqrt{16})$ & $d(w ; \beta)$ from[17) \\
\hline 1 & 30 & 0.016040 & 0.027472 \\
\hline 5 & 10 & 0.012639 & 0.018288 \\
\hline 6 & 100 & 0.001068 & 0.002247 \\
\hline 14 & 5 & 0.009564 & 0.013908 \\
\hline 50 & 100 & 0.000137 & 0.000343 \\
\hline 500 & 1000 & $1.38 \times 10^{-6}$ & $5.02 \times 10^{-6}$ \\
\hline 1000 & 5000 & $1.3 \times 10^{-7}$ & $5.8 \times 10^{-7}$ \\
\hline
\end{tabular}

TABLE I

BOUNDS FOR $d(w ; \beta)$ COMPUTED By 16 AND 17, RESPECTIVELY

The numerical results are plotted in Fig. 3 (for the case $\beta=5, w=3 ; d=0.0398921)$ and Fig. 4 (for the case $\beta=10, w=4 ; d=0.0154697$ ).

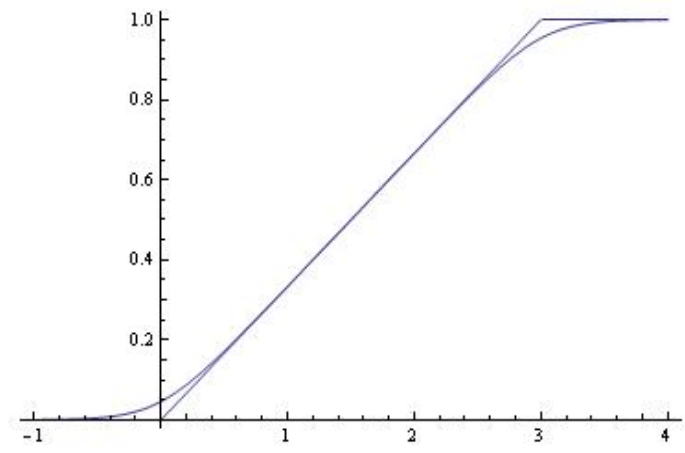

Fig. 3. Functions $c_{\delta, \delta}$ and $s_{\delta, \delta}^{(\beta)}$ for $\beta=5, w=3$; $d \leq 0.4$.

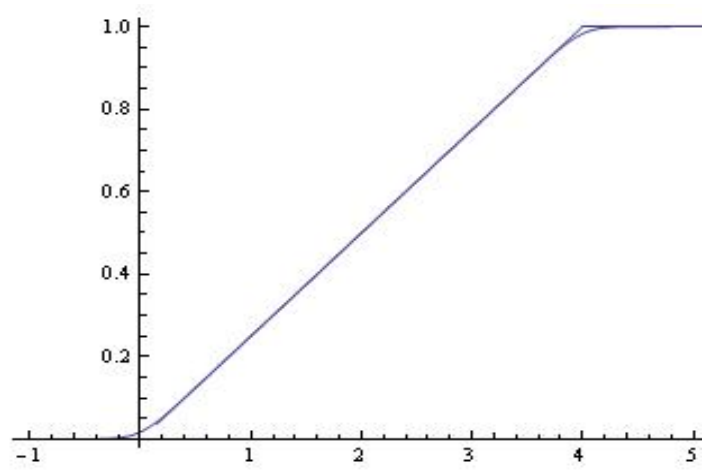

Fig. 4. Functions $c_{\delta, \delta}$ and $s_{\delta, \delta}^{(\beta)}$ for $\beta=10, w=4 ; d \leq$ 0.016 .

\section{APPROXIMATION OF THE STEP FUNCTION BY THE GOMPERTZ FUNCTION}

In this section we study the Hausdorff approximation of the step function by the Gompertz function and obtain precise upper and lower bounds for the Hausdorff distance. Numerical examples, illustrating our results are given.

Definition 4. The Gompertz function $\sigma_{\alpha, \beta}(t)$ is defined for $\alpha, \beta>0$ by [22]:

$$
\sigma_{\alpha, \beta}(t)=e^{-\alpha e^{-\beta t}} \text {. }
$$

Special case 3. For $\alpha^{*}=\ln 2=0.69314718 \ldots$ we obtain the special Gompertz function:

$$
\sigma_{\alpha^{*}, \beta}(t)=e^{-\alpha^{*} e^{-\beta t}}
$$

such that $\sigma_{\alpha^{*}, \beta}(0)=1 / 2$. 
A. Iliev et al., On the Approximation of the Cut and Step Functions by Logistic ...

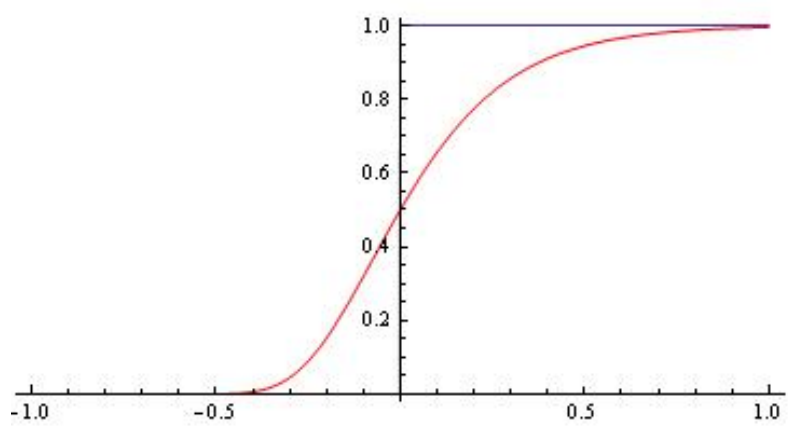

Fig. 5. The Gompertz function with $\alpha=\ln 2$ and $\beta=5$; H-distance $d=0.212765$.

We study the Hausdorff approximation of the Heaviside step function $c_{0}=h_{0}(t)$ by Gompertz functions of the form (18) and find an expression for the error of the best approximation.

The H-distance $d=d\left(\alpha^{*}, \beta\right)$ between the Heaviside step function $h_{0}(t)$ and the Gompertz function (19) satisfies the relation

$$
\sigma_{\alpha^{*}, \beta}(d)=e^{-\alpha^{*} e^{-\beta d}}=1-d
$$

or

$$
\ln (1-d)+\alpha^{*} e^{-\beta d}=0 .
$$

The following theorem gives upper and lower bounds for $d\left(\alpha^{*}, \beta\right)$. For brevity we denote $\alpha=\alpha^{*}$ in Theorem 1 and its proof.

Theorem 1. The Hausdorff distance $d=d(\alpha, \beta)$ between the step function $h_{0}$ and the Gompertz function (19) can be expressed in terms of the parameter $\beta$ for any real $\beta \geq 2$ as follows:

$$
\frac{2 \alpha-1}{1+\alpha \beta}<d<\frac{\ln (1+\alpha \beta)}{1+\alpha \beta} .
$$

Proof. We need to express $d$ in terms of $\alpha$ and $\beta$, using 20. Let us examine the function $F(d)=$ $\ln (1-d)+\alpha e^{-\beta d}$. From

$$
F^{\prime}(d)=-\frac{1}{1-d}-\alpha \beta e^{-\beta d}<0
$$

we conclude that the function $F$ is strictly monotone decreasing. Consider function $G(d)=\alpha-$ $(1+\alpha \beta) d$. From Taylor expansion

$$
\alpha-(1+\alpha \beta) d-\ln (1-d)-\alpha e^{-\beta d}=O\left(d^{2}\right)
$$

we obtain $G(d)-F(d)=\alpha-(1+\alpha \beta) d-\ln (1-$ $d)-\alpha e^{-\beta d}=O\left(d^{2}\right)$. Hence $G(d)$ approximates $F(d)$ with $d \rightarrow 0$ as $O\left(d^{2}\right)$. In addition $G^{\prime}(d)=$ $-(1+\alpha \beta)<0$. Further, for $\beta \geq 2$,

$$
\begin{aligned}
G\left(\frac{2 \alpha-1}{1+\alpha \beta}\right) & =1-\alpha>0, \\
G\left(\frac{\ln (1+\alpha \beta)}{1+\alpha \beta}\right) & =\alpha-\ln (1+\alpha \beta)<0 .
\end{aligned}
$$

This completes the proof of the theorem.

Some computational examples using relation 20) are presented in Table 2.

\begin{tabular}{|c|c|}
\hline$\beta$ & $d\left(\alpha^{*}, \beta\right)$ \\
\hline 2 & 0.310825 \\
5 & 0.212765 \\
10 & 0.147136 \\
50 & 0.0514763 \\
100 & 0.0309364 \\
500 & 0.00873829 \\
1000 & 0.00494117 \\
\hline
\end{tabular}

TABLE II

BOUNDS FOR $d\left(\alpha^{*}, \beta\right)$ COMPUTED BY 20 FOR VARIOUS $\beta$.

The calculation of the value of the H-distance between the Gompertz sigmoid function and the Heaviside step function is given in Appendix 1.

The numerical results are plotted in Fig. 5 (for the case $\alpha^{*}=\ln 2, \beta=5$, H-distance $d=0.212765$ ) and Fig. 6 (for the case $\alpha^{*}=\ln 2$, $\beta=20$, H-distance $d=0.0962215$ ).

Remark 1. For some comparisons of the Gompertz and logistic equation from both practical and theoretical perspective, see [6], [8], [40]. As can be seen from Figure 6 the graph of the Gompertz function is "skewed", it is not symmetric with respect to the inflection point. In biology, the Gompertz function is commonly used to model growth process where the period of increasing growth is shorter than the period in which growth decreases [8], [33]. 
A. Iliev et al., On the Approximation of the Cut and Step Functions by Logistic ...

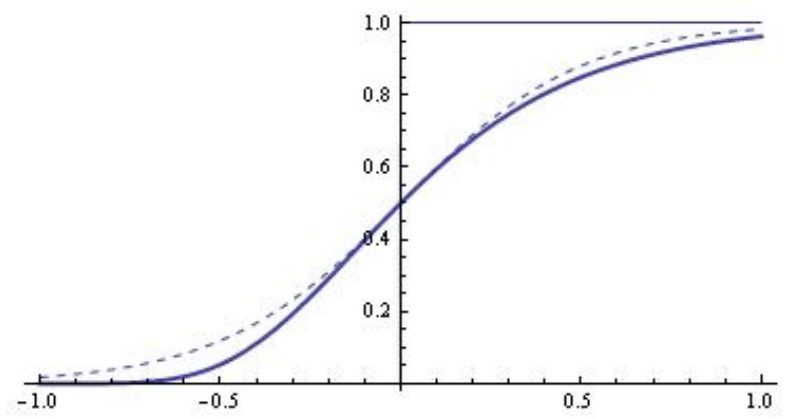

Fig. 6. The logistic (dotted line) and the Gompertz function (dense line) with same point and same rate (at that point).

Remark 2. For $k>0, \beta>0$ consider the differential equation

$$
y^{\prime}=k e^{-\beta t} y, \quad \frac{k}{\beta}=\alpha .
$$

We have

$$
\begin{gathered}
\frac{d y}{d t}=k e^{-\beta t} y ; \quad \frac{d y}{y}=k e^{-\beta t} d t \\
\ln y=-\frac{k}{\beta} e^{-\beta t}=-\alpha e^{-\beta t} ; \quad y=e^{-\alpha e^{-\beta t}} .
\end{gathered}
$$

We see that the solution of differential equation [22 is the Gompertz function $\sigma_{\alpha, \beta}(t)$ [18] [6]). As shown in [28], equation (22) can be interpreted as $y^{\prime}=k s y$, wherein $s=s(t)$ is the nutrient substrate used for the growth of the population; one see that $s$ is a decay exponential function in the Gompertz model (a similar interpretation can be found in [21]), [40]). For other interpretations see [6]), [8], [20].

\section{CONCLUSION}

In this paper we discuss several computational, modelling and approximation issues related to two familiar classes of sigmoid functions - the logistic (Verhulst) and the Gompertz functions. Both classes find numerous applications in various fields of life sciences, ecology, medicine, artificial neural networks, fuzzy set theory, etc.

\section{bigskip}

We study the uniform and Hausdorff approximation of the cut functions by logistic functions. We demonstrate that the best uniform approximation between a cut function and the respective logistic function is an absolute constant not depending on the (largest) slope $k$. On the other side we show that the Hausdorff distance (H-distance) depends on the slope $k$ and tends to zero with $k \rightarrow \infty$. We also discuss the limiting case when the cut function tends to the Heaviside step function in Hausdorff sense, thereby extending a related previous result [4].

The approximation of the cut function by a family of squashing functions induced by the logistic function is also discussed. We propose a new estimate for the H-distance between a cut function and its best approximating squashing function. Our estimate extends a known result stating that the cut function can be approximated arbitrary well by squashing functions [18]. Our estimate is also extended to cover the limiting case of the Heaviside step function.

Finally we study the approximation of the cut and step functions by the family of Gompertz functions. New estimates for the $\mathrm{H}$-distance between a cut function and its best approximating Gompertz function are obtained.

\section{REFERENCES}

[1] A. Akanuma, Parameter Analysis of Gompertz Function Growth Model in Clinical Tumors, European J. of Cancer 14 (1978) 681-688.

[2] G. Albano and V. Giono, On the First Exit Time Problem for a Gompertz-type Tumor Growth, Lecture Notes in Computer Science 5717 (2009) 113-120, http://dx.doi.org/10.1007/978-3-642-04772-5_16

[3] R. Alt and S. Markov, Theoretical and Computational Studies of some Bioreactor Models, Computers and Mathematics with Applications 64(3) (2012) 350-360, http://dx.doi.org/10.1016/j.camwa.2012.02.046

[4] R. Anguelov and S. Markov, Hausdorff Continuous Interval Functions and Approximations, LNCS (SCAN 2014 Proceedings), to appear.

[5] I. A. Basheer and M. Hajmeer, Artificial Neural Networks: Fundamentals, Computing, Design, and Application, Journal of Microbiological Methods 43(1) (2000) 3-31, http://dx.doi.org/10.1016/S0167-7012(00)00201-3

[6] Z. Bajzer and S. Vuk-Pavlovic, New Dimensions in Gompertz Growth, J. of Theoretical Medicine 2(4) (2000) 307-315, http://dx.doi.org/10.1080/10273660008833057 
[7] D. E. Bentila, B. M. Osei, C. D. Ellingwood and J. P. Hoffmann, Analysis of a Schnute Postulate-based Unified Growth Mode for Model Selection in Evolutionary Computations, Biosystems 90(2) (2007) 467-474, http://dx.doi.org/10.1016/j.biosystems.2006.11.006

[8] R. D. Berger, Comparison of the Gompertz and Logistic Equation to Describe Plant Disease Progress, Phytopathology 71 (1981) 716-719, http://dx.doi.org/10.1094/Phyto-71-716

[9] M. Carrillo and J. M. Gonzalez, A New Approach to Modelling Sigmoidal Curves, Technological Forecasting and Social Change 69(3) (2002) 233-241, http://dx.doi.org/10.1016/S0040-1625(01)00150-0

[10] M. E. Cayre, G. Vignolob and O. Garroa, Modeling Lactic Acid Bacteria Growth in Vacuum-packaged Cooked Meat Emulsions Stored at Three Temperatures, Food Microbiology 20(5) (2003) 561-566, http://dx.doi.org/10.1016/S0740-0020(02)00154-5

[11] Y. Chalco-Cano, H. Roman-Flores and F. Gomida, A New Type of Approximation for Fuzzy Intervals, Fuzzy Sets and Systems 159(11) (2008) 1376-1383, http://dx.doi.org/10.1016/j.fss.2007.12.025

[12] Z. Chen and F. Cao, The Approximation Operators with Sigmoidal Functions, Computers \& Mathematics with Applications 58(4) (2009) 758-765, http://dx.doi.org/10.1016/j.camwa.2009.05.001

[13] Z. Chen and F. Cao, The Construction and Approximation of a Class of Neural Networks Operators with Ramp Functions, Journal of Computational Analysis and Applications 14(1) (2012) 101-112.

[14] Z. Chen, F. Cao and J. Hu, Approximation by Network Operators with Logistic Activation Functions, Applied Mathematics and Computation 256 (2015) 565-571, http://dx.doi.org/10.1016/j.amc.2015.01.049

[15] E. S. Chumerina, Choice of Optimal Strategy of Tumor Chemotherapy in Gompertz. Model, J. Comp. and Syst. Sci. Int. 48(2) (2009) 325-331, http://dx.doi.org/10.1134/S1064230709020154

[16] D. Costarelli and R. Spigler, Approximation Results for Neural Network Operators Activated by Sigmoidal Functions, Neural Networks 44 (2013) 101-106, http://dx.doi.org/10.1016/j.neunet.2013.03.015

[17] D. Costarelli and R. Spigler, Constructive Approximation by Superposition of Sigmoidal Functions, Anal. Theory Appl. 29(2) (2013) 169-196, http://dx.doi.org/10.4208/ata.2013.v29.n2.8

[18] J. Dombi and Z. Gera, The Approximation of Piecewise Linear Membership Functions and Lukasiewicz Operators, Fuzzy Sets and Systems 154(2) (2005) 275-286, http://dx.doi.org/10.1016/j.fss.2005.02.016

[19] H. Enderling and M. A. J. Chaplain, Mathematical Modeling of Tumor Growth and Treatment, Curr. Pharm. Des. 20(30) (2014) 4934-4940, http://dx.doi.org/10.2174/1381612819666131125150434

[20] R. I. Fletcher, A General Solution for the Complete Richards Function, Mathematical Biosciences 27(3-4)
(1975) 349-360,

http://dx.doi.org/10.1016/0025-5564(75)90112-1

[21] J. France, J. Dijkstra and M. S. Dhanoa, Growth Functions and Their Application in Animal Science, Annales de Zootechnie 45(Suppl 1) (1996) 165-174.

[22] B. Gompertz, On the Nature of the Function Expressive of the Law of Human Mortality, and on a New Mode of Determining the Value of the Life Contingencies, Philos. Trans. R. Soc. London 115 (1825) 513-585.

[23] J. Han and C. Morag, The Influence of the Sigmoid Function Parameters on the Speed of Backpropagation Learning, In: Mira, J., Sandoval, F. (Eds) From Natural to Artificial Neural Computation 930 (1995) 195-201, http://dx.doi.org/10.1007/3-540-59497-3_175

[24] F. Hausdorff, Set Theory (2 ed.), New York, Chelsea Publ. (1962 [1957]) (Republished by AMS-Chelsea 2005), ISBN: 978-0-821-83835-8.

[25] M. Kodaka, Requirements for Generating Sigmoidal Time-course Aggregation in Nucleation-dependent Polymerization Model, Biophys. Chem. 107(3) (2004) 243253, http://dx.doi.org/10.1016/j.bpc.2003.09.013

[26] M. Kodaka, Interpretation of Concentration-dependence in Aggregation Kinetics, Biophys. Chem. 109(2) (2004) 325-332, http://dx.doi.org/10.1016/j.bpc.2003.12.003

[27] M. Koivula, M. Sevon-Aimonen, I. Stranden, K. Matilainen, T. Serenius, K. Stalder and E. Mantysaari, Genetic (Co)Variances and Breeding Value Estimation of Gompertz Growth Curve Parameters in Finish Yorkshire Boars, Gilts and Barrows, J. Anim. Breed. Genet. 125(3) (2008) 168-175, http://dx.doi.org/10.1111/j.1439-0388.2008.00726.x

[28] N. Kyurkchiev and S. Markov, Sigmoidal Functions: Some Computational and Modelling Aspects, Biomath Communications 1(2), (2014), http://dx.doi.org/10.11145/j.bmc.2015.03.081

[29] N. Kyurkchiev and S. Markov, On the Hausdorff Distance Between the Heaviside Step Function and Verhulst Logistic Function, J. Math. Chem., to appear.

[30] N. Kyurkchiev and S. Markov, Sigmoid Functions: Some Approximation and Modelling Aspects. Some Moduli in Programming Environment Mathematica, LAP (Lambert Acad. Publ.) (2015), ISBN: 978-3-659-76045-7.

[31] T. P. Labuza and B. Fu, Growth Kinetics for Shelf-life Prediction: Theory and Practice, Journal of Industrial Microbiology 12(3-5) (1993) 309-323, http://dx.doi.org/10.1007/BF01584208

[32] A. K. Laird, Dynamics of Tumor Growth, Br. J. Cancer 18(3) (1964) 490-502.

[33] D. Lin, Z. Shkedy, D. Yekutieli, D. Amaratunda and L. Bijnens (Eds.), Modeling Dose Responce Microarray Data in Early Drug Development Experiments Using $R$, Springer (2012), ISBN: 978-3-642-24006-5.

[34] S. Markov, Cell Growth Models Using Reaction Schemes: Batch Cultivation, Biomath 2(2) (2013), 1312301 , http://dx.doi.org/10.11145/j.biomath.2013.12.301

[35] A. G. McKendrick and M. Kesava Pai, The Rate of Mul- 
A. Iliev et al., On the Approximation of the Cut and Step Functions by Logistic ...

tiplication of Micro-organisms: A Mathematical Study, Proc. of the Royal Society of Edinburgh 31 (1912) 649653, http://dx.doi.org/10.1017/S0370164600025426

[36] N. Radchenkova, M. Kambourova, S. Vassilev, R. Alt and S. Markov, On the Mathematical Modelling of EPS Production by a Thermophilic Bacterium, Biomath 3(1) (2014), 1407121, http://dx.doi.org/10.11145/j.biomath.2014.07.121

[37] F. J. Richards, A Flexible Growth Function for Empirical Use, J. Exp. Bot. 10 (1959) 290-300, http://dx.doi.org/10.1093/jxb/10.2.290

[38] R. Rickles and A. Scheuerlein, Biological Imlications of the Weibull and Gompertz Models of Aging, J. of Gerontology: Biologica Sciences 57(2) (2002) B69-B76, http://dx.doi.org/10.1093/gerona/57.2.B69

[39] A. Sas, H. Snieder and J. Korf, Gompertz' Survivorship Law as an Intrinsic Principle of Aging, Medical Hypotheses 78(5) (2012) 659-663, http://dx.doi.org/10.1016/j.mehy.2012.02.004

[40] M. A. Savageau, Allometric Morphogenesis of Complex Systems: Derivation of the Basic Equations from the First Principles, Proc. of the National Academy of Sci. USA 76(12) (1979) 6023-6025.

[41] B. Sendov, Hausdorff Approximations, Kluwer (1990), ISBN: 978-94-010-6787-4, e-ISBN: 978-94-0090673-0, http://dx.doi.org/10.1007/978-94-009-0673-0

[42] C. J. Stannard, A. P. Williams and P. A. Gibbs, Temperature/growth Relationship for Psychrotrophic Foodspoilage Bacteria, Food Microbiol. 2(2) (1985) 115-122, http://dx.doi.org/10.1016/S0740-0020(85)80004-6

[43] J. H. Van der Walt, The Linear Space of Hausdorff Continuous Interval Functions, Biomath 2(2) (2013), 1311261, http://dx.doi.org/10.11145/j.biomath.2013.11.261

[44] P.-F. Verhulst, Notice Sur la Loi Que la Population Poursuit dans Son Accroissement, Correspondance Mathematique et Physique 10 (1838) 113-121.

[45] P.-F. Verhulst, Recherches Mathematiques sur la Loi D'accroissement de la Population (Mathematical Researches into the Law of Population Growth Increase), Nouveaux Memoires de l'Academie Royale des Sciences et Belles-Lettres de Bruxelles 18 (1845) 1-42.

[46] P.-F. Verhulst, Deuxieme Memoire sur la Loi D'accroissement de la Population, Memoires de l'Academie Royale des Sciences, des Lettres et des Beaux-Arts de Belgique 20 (1847) 1-32.

[47] D. L. Wilson, The Analysis of Survival (Mortality) Data: Fitting Gompertz, Weibull, and Logistic Functions, Mech. Ageing Devel. 74(1-2) (1994) 15-33, http://dx.doi.org/10.1016/0047-6374(94)90095-7

[48] C. P. Winsor, The Gompertz Curve as a Growth Curve, Proceedings of the National Academy of Sciences 18(1) (1932) 1-8.

[49] M. H. Zwietering, I. Jongenburger, F. M. Rombouts and K. van't Riet, Modeling of the Bacterial Growth Curve, Appl. Envir. Microbiol. 56(6) (1990) 1875-1881.

[50] M. H. Zwietering, H. G. Cuppers, J. C. de Wit and
K. van't Riet, Evaluation of Data Transformations and Validation of a Model for the Effect of Temperature on Bacterial Growth, Appl. Environ. Microbiol. 60(1) (1994) 195-203. 
A. Iliev et al., On the Approximation of the Cut and Step Functions by Logistic ...

\section{APPENDIX}

The Module "Computation of the distance $d$ and visualization of the cut function $c_{\Delta}$ and squashing function $s_{\Delta}^{(\beta)}$ " in CAS MATHEMATICA. 
A. Iliev et al., On the Approximation of the Cut and Step Functions by Logistic ...

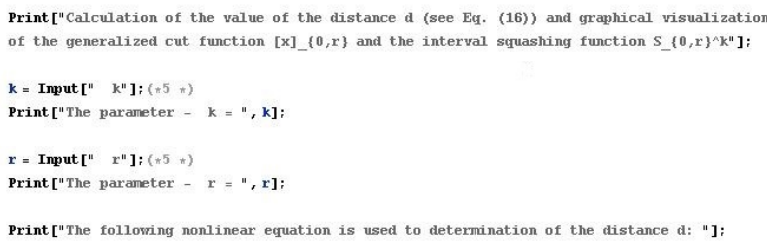

Fig. 7. Module in programming environment MATHEMATICA.

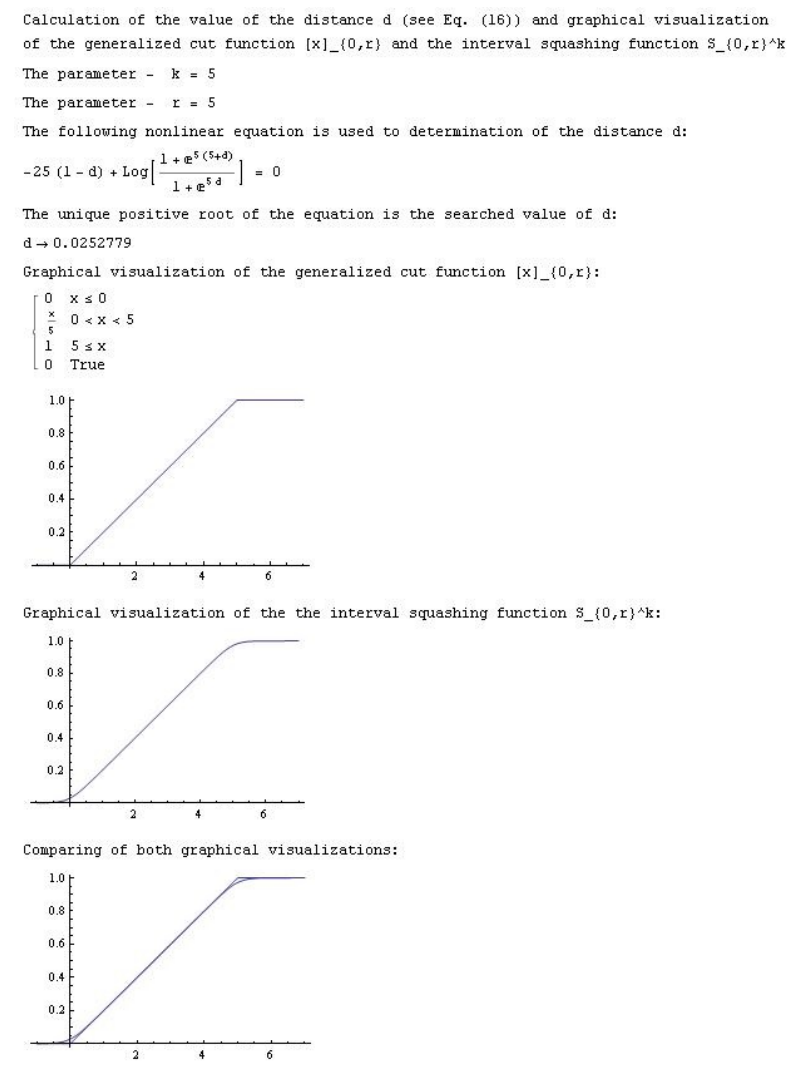

Fig. 8. The test provided on our control example. 